\title{
Optimización de la Producción de Biocrudo mediante Licuefacción Hidrotérmica Catalizada de Buchón de Agua
}

\author{
Elkin A. Gómez, Alexander Osorio, David Ocampo y Luis A. Ríos* \\ Procesos Químicos Industriales, Departamento de Ingeniería Química, Facultad de Ingeniería, Universidad de Antioquia \\ U de A, Calle 70 No. 52-21, Medellín, Colombia. (e-mail: elkina.gomez@udea.edu.co; alexander.osoriog@udea.edu.co; \\ david.ocampoe@udea.edu.co; luis.rios@udea.edu.co)
}

* Autor a quien debe ser dirigida la correspondencia.

Recibido Oct. 24, 2018; Aceptado Ene. 4, 2019; Versión final Mar. 4, 2019, Publicado Oct. 2019

\section{Resumen}

El objetivo del trabajo fue llevar a cabo la evaluación del proceso de licuefacción con biomasa de Buchón de Agua. Para esto se desarrolló un diseño experimental factorial con un modelo cúbico, determinando el efecto de las variables temperatura, tiempo y relación líquido/sólido (L/S) sobre la variable respuesta Rendimiento Energético (RE). Los mejores resultados se obtuvieron a $300^{\circ} \mathrm{C}, 60$ min y $\mathrm{L} / \mathrm{S}=10$ (exp. 15) con $\mathrm{RE}$ de 6,34 $\mathrm{MJ} / \mathrm{kg}$ biomasa, a $300^{\circ} \mathrm{C}, 30 \mathrm{~min}$ y $\mathrm{L} / \mathrm{S}=10$ (exp. 5) con $\mathrm{RE}$ de $6,31 \mathrm{MJ} / \mathrm{kg}$ biomasa y a $270^{\circ} \mathrm{C}, 60 \mathrm{~min}$ y $\mathrm{L} / \mathrm{S}=20$ (exp. 9) con 6,29 MJ/kg biomasa, seleccionándose las condiciones del exp. 5 como las más adecuadas dada la menor relación L/S y el menor tiempo de reacción. La ventaja de adicionar catalizador se hizo evidente al comparar los resultados del RE del experimento 26 con catalizador (5,9 MJ/kg biomasa) y del experimento sin catalizador (3,3 MJ/kg biomasa) bajo las mismas condiciones. Se pudo determinar además que los biocrudos se componían principalmente de compuestos aromáticos, ácidos grasos, hidrocarburos alifáticos, cetonas, aldehídos y alcoholes, y ácidos carboxílicos a condiciones más drásticas.

\section{Optimization of Biooil Production by Catalytic Hydrothermal Liquefaction of Water Hyacinth}

\begin{abstract}
The main objective of this paper was to carry out the evaluation of the liquefaction process on Water Hyacinth biomass. To achieve this, a factorial experimental design with a cubic model was developed, for determining the effect of the variables temperature, time and liquid/solid ratio (L/S) on the response variable Energy Yield (EY). Best results were obtained at $300^{\circ} \mathrm{C}, 60 \mathrm{~min}$ and $\mathrm{L} / \mathrm{S}=10$ (exp. 15) with $\mathrm{RE}$ of $6.34 \mathrm{MJ} / \mathrm{kg}$ biomass, at $300^{\circ} \mathrm{C}, 30 \mathrm{~min}$ and $\mathrm{L} / \mathrm{S}=10$ (exp. 5) with EY of $6.31 \mathrm{MJ} / \mathrm{kg}$ biomass and at $270^{\circ} \mathrm{C}, 60 \mathrm{~min}$ and $\mathrm{L} / \mathrm{S}=20$ (exp. 9) with $6.29 \mathrm{MJ} / \mathrm{kg}$ biomass, selecting the conditions of exp. 5 as the most appropriate given the lower L/S ratio and the shorter reaction time. The advantage of catalyst presence in the reaction was evident when comparing the results of the EY in exp. 26 with catalyst (5.9 MJ/kg biomass) and experiment without catalyst (3.3 MJ / kg biomass) under the same conditions. It was also possible to determine that the biooils were composed mainly of aromatic compounds, fatty acids, aliphatic hydrocarbons, ketones, aldehydes and alcohols, and carboxylic acids under more drastic conditions.
\end{abstract}

Keywords: biofuels; biooil; water hyacinth; hydrothermal liquefaction 


\section{INTRODUCCIÓN}

El exagerado aporte de nutrientes a los sistemas acuáticos por malas prácticas en actividades agrícolas, domésticas e industriales ha ocasionado que a nivel mundial gran parte de las lagunas, lagos, canales y embalses sufran procesos de eutrofización y, por lo tanto, presencia masiva de algas y plantas acuáticas, causando impactos significativos en los procesos físicos, químicos y bióticos de los ecosistemas. El Buchón de Agua (Eichhornia crassipes) es una especie invasora que por su alta capacidad adaptativa y reproductiva ocupa el puesto 8 entre las 10 malezas más invasivas del mundo (Arteaga et al., 2010). En Sudamérica cerca del $41 \%$ de los lagos sufren este problema (Tedesco, 2009). Tan solo en la represa de Porce II (Antioquia Colombia) se recogieron en 2010 1'410.187 toneladas de Buchón de Agua (Arteaga et al., 2010). Esta alta productividad puede pasar de ser un problema ambiental a un material energético promisorio.

El proceso de obtención de energía y combustibles a partir de la licuefacción hidrotérmica de la biomasa ha sido considerado en las últimas décadas como una de las tecnologías que puede llegar a suplir parte de la demanda energética futura mediante una estrategia ambiental y económicamente viable. Esta tecnología tiene la gran ventaja de no requerir el secado de la biomasa, con lo cual se reduce el enorme gasto energético de evaporar agua, que se requiere otras tecnologías como la pirolisis y la gasificación. Zhang et al. (Zhang, et al., 2013a) investigaron la licuefacción hidrotérmica de Buchón de Agua con el fin de analizar la distribución de productos e identificarlos. Las reacciones fueron llevadas a cabo entre $240^{\circ} \mathrm{C}$ y $340^{\circ} \mathrm{C}$ por 10 min y $\sin$ catalizador, en un reactor de $250 \mathrm{~mL}$. Los análisis cromatográficos (GC - MS) del el biocrudo pesado obtenido mostraron que a temperaturas de $240^{\circ} \mathrm{C}$ y $260^{\circ} \mathrm{C}$ se obtienen los compuestos de menor peso molecular, incluyendo ésteres y furanos, mientras que a $280^{\circ} \mathrm{C}$ se obtienen mayormente ácidos grasos. Entre $300^{\circ} \mathrm{C}$ y $340^{\circ} \mathrm{C}$ la distribución de productos se hizo más compleja, con presencia de alcanos, fenoles y pirroles, desapareciendo los furanos debido a su alta inestabilidad a las condiciones de la licuefacción. Respecto a los análisis llevados a cabo sobre el biocrudo soluble en agua, los resultados cromatográficos mostraron una composición consistente principalmente en cetonas, ácidos grasos y aldehídos, resultantes de la descomposición de la celulosa y hemicelulosa, así como también, pirazinas y piridinas y otros compuestos heterocíclicos conteniendo nitrógeno. Por encima de los $280^{\circ} \mathrm{C}$ se obtuvieron también fenoles y ciclopentenos. Respecto al poder calorífico (HHV), se vio incrementado al aumentar la temperatura de reacción, obteniéndose el mayor de $33,83 \mathrm{MJ} / \mathrm{Kg}$ a $340^{\circ} \mathrm{C}$. El mayor rendimiento de biocrudo pesado, $20 \%$, se obtuvo a $320^{\circ} \mathrm{C}$ (Zhang et al., 2013b). Desafortunadamente, los efectos de las variables tiempo, relación líquido/sólido y presencia de catalizador en el proceso de licuefacción no fueron determinados.

Singh y colaboradores (Singh et al., 2015) también estudiaron la licuefacción del buchón de agua empleando $\mathrm{KOH}$ y K2CO3 como catalizadores, con tiempos de reacción entre 15 y 60 min. La relación biomasa-agua también fue variada, $1: 3,1: 6$ y $1: 12$ y temperaturas de 250,280 y $300^{\circ} \mathrm{C}$. El mayor rendimiento de biocrudo total, $23 \%$, fue obtenido con una solución de $\mathrm{KOH} 1 \mathrm{~N}$ como catalizador, a $280^{\circ} \mathrm{C}$, tiempo de reacción de 15 min y con una relación líquido/biomasa de $6 \mathrm{~g} / \mathrm{g}$. Los análisis FTIR mostraron que los biocrudos se componían principalmente de ácidos carboxílicos y sus ésteres derivados, fenoles, compuestos aromáticos policíclicos y ésteres aromáticos. A los biocrudos también les fueron realizador análisis HNMR y CNMR que indicaron altos porcentajes de grupos funcionales alifáticos, efecto que se acentuó con el uso de catalizadores alcalinos (Singh et al., 2015). El estudio se centró principalmente en la caracterización del biocrudo y en determinar que con la adición de catalizadores alcalinos ( $\mathrm{KOH}$ y K2CO3) se incrementa el rendimiento hacia biocrudo. Es importante destacar que no se determinaron los valores para el poder calorífico de los biocrudos, el cual es una de las variables respuestas más importantes para este tipo de estudios, pues un aparentemente alto rendimiento a biocrudo puede no ser tan buen resultado si este tiene un muy alto contenido en oxígeno y por lo tanto un bajo poder calorífico. Un alto contenido en oxígeno hace que el rendimiento final del biocombustible se reduzca mucho, puesto que el oxígeno debe ser eliminado del biocombustible, mediante reacciones de hidrodeoxigenación, para aumentarle su poder calorífico; además, se aumentan los costos de procesamiento asociados a la hidrodeoxigenación del biocombustible (consumo de hidrógeno y tiempos de reacción). Por otro lado, Singh et al. Tampoco investigaron el efecto sinérgico de las tres variables, temperatura, tiempo de reacción y relación líquido/ sólido.

El Buchón de Agua también ha sido estudiado mediante procesos de pirólisis. Biswas et al. llevaron a cabo pirolisis de biomasa de Buchón de Agua en un reactor de vidrio de lecho fijo entre 300 y $450^{\circ} \mathrm{C}$, encontrando el mayor rendimiento de biocrudo $(24,6 \%)$ a $400^{\circ} \mathrm{C}$. El biocrudo se obtuvo luego de condensar los gases de reacción y posteriormente el agua en el biocrudo se removió empleando sulfato de sodio anhidro y la fracción orgánica se extrajo usando diétil éter. Los espectros GC-MS, HNMR, and FT-IR del biocrudo mostraron un alto porcentaje de grupos funcionales alifáticos y presencia de grupos fenólicos, cetonas y nitrogenados (Biswas et al., 2017). 
Respecto a otras biomasas, la licuefacción hidrotérmica ha sido ampliamente estudiada. Biomasas maderables, biomasa microalgal y residuos agroindustriales como los provenientes de la industria de la palma de aceite han sido sometidas al proceso de licuefacción hidrotérmica. Osorio et al., (2019) llevaron a cabo licuefacción de madera de acacia usando agua como solvente en un reactor Parr observando el efecto de la temperatura, tiempo de reacción y relación solvente (agua)/biomasa. En su estudio los investigadores encontraron como mejores condiciones de operación $330^{\circ} \mathrm{C}$, una relación solvente (agua)/biomasa de15 y un tiempo de reacción de 0 minutos para obtener rendimiento másico del $36 \%$ y poder calorífico de $31,7 \mathrm{MJ} / \mathrm{kg}$. En Malasia se estudió la licuefacción de racimos vacíos de la palma de aceite con catalizadores basados en Zn. El catalizador con una carga de $15 \%$ peso de Zn soportado en una zeolita ZSM-5 mostró el mayor rendimiento de biocrudo después de la introducción del catalizador. Asimismo, estudiaron la influencia de otros parámetros en el proceso de licuefacción como la temperatura de reacción, el tiempo de reacción, la composición del catalizador y la carga del catalizador, encontrando una conversión óptima de $43,6 \%$ a $180^{\circ} \mathrm{C}$, 90 min de reacción con 0,5\% peso de Zn soportado en ZSm-5 (Rachel-Tang et al., 2017).

Algunas investigaciones se han centrado en el análisis del efecto del catalizador sobre el rendimiento de biocrudo. Este es el caso del estudio llevado a cabo por Yim et al. en 2017 en el que se catalizó la licuefacción hidrotérmica supercrítica de racimos vacíos de palma de aceite con $\mathrm{CaO}, \mathrm{MgO}, \mathrm{MnO}, \mathrm{ZnO}, \mathrm{NiO}, \mathrm{SnO}, \mathrm{CeO}_{2}$, $\mathrm{Al}_{2} \mathrm{O}_{3}$ y $\mathrm{La}_{2} \mathrm{O}_{3}$. Las reacciones fueron llevadas a cabo con cargas de catalizador de $1,0 \%$ peso y con temperatura de reacción de $390^{\circ} \mathrm{C}, 25 \mathrm{MPa}$ de presión por $1 \mathrm{~h}$. Entre los catalizadores empleados se encontró que $\mathrm{La}_{2} \mathrm{O}_{3}, \mathrm{CeO}_{2}, \mathrm{MnO}$ y $\mathrm{CaO}$ fueron los más activos en la licuefacción, obteniendo como mayor rendimiento de biocrudo la reacción llevada a cabo con $\mathrm{CeO}_{2}$ (1,44 veces el rendimiento que sin usar catalizador). El análisis CG-MS y FT-IR mostró que los productos se componían en su mayor parte por compuestos fenólicos, cetonas, ácidos carboxílicos y otros compuestos aromáticos.

En este artículo se presentan resultados obtenidos en la licuefacción de biomasa de Buchón de Agua y el efecto de las variables temperatura, relación líquido/sólido y tiempo de reacción sobre la producción de biocrudo en rangos de temperatura entre $270-330^{\circ} \mathrm{C}$, relaciones liquido/sólido 10/1, 15/1 y 20/1, y tiempos de reacción de 0,30 y 60 min, con el fin de determinar las mejores condiciones para la obtención de un biocrudo con una alta disponibilidad energética, definida por la variable respuesta Rendimiento Energético (energía contenida en el biocrudo que se obtiene por cada $1 \mathrm{Kg}$ de biomasa alimentada).

\section{METODOLOGÍA}

La metodología se divide en cuatro subsecciones: materiales, experimentación, separación de los productos de reacción y caracterización de productos.

\section{Materiales}

Buchón de Agua del municipio de Porce (Antioquia - Colombia) fue utilizado como materia prima en la evaluación de la licuefacción hidrotérmica. El material fue sometido un proceso de secado a temperatura de $70 \stackrel{\circ}{\circ}$ hasta lograr una humedad inferior al 10\%, para evitar su degradación biológica durante el almacenamiento. También se le efectuó un proceso de conminución mecánica en un molino vegetal de cuchillas y a tamizaje con el fin de homogenizar su distribución de tamaño de partícula [0,833-1397 $\mu \mathrm{m}]$.

\section{Experimentación}

Se evaluó un diseño experimental factorial 33 con el fin de evaluar variables como temperatura, relación biomasa solvente y tiempo de reacción. Como catalizador se utilizó el carbonato de potasio K2CO3 (Mazaheri et al., 2013; Xu et al., 2008) y agua como solvente. En la Tabla 1 se muestran las condiciones experimentales evaluadas para la licuefacción de Buchón de Agua, las cuales se establecieron a partir de literatura existente (Anastasakis et al., 2011; Demirbaş, 2008; Singh et al., 2015; Zhang et al., 2013b).

Tabla 1: Condiciones experimentales de la licuefacción hidrotérmica de las biomasas de referencia empleadas.

\begin{tabular}{lr}
\hline \multicolumn{1}{c}{ Variable } & \multicolumn{1}{c}{ Valor } \\
\hline Presión inicial de Ar & 10 bar \\
Temperatura & $270,300,330 \stackrel{\circ}{C}$ \\
Tiempo & $0,30,60$ minutos \\
Tamaño de partícula & $833-1397 \mu \mathrm{m}$ \\
\hline
\end{tabular}


Tabla 1 (continuación)

\begin{tabular}{lr}
\hline Solvente & agua \\
Catalizador & $\mathrm{K}_{2} \mathrm{CO}_{3}$ \\
Carga de catalizador & $10 \%(\mathrm{w} / \mathrm{w})$ respecto a la biomasa \\
Atmósfera & Argón \\
Cantidad de biomasa & $6 \mathrm{~g}$ \\
Relación líquido/sólido & $10 / 1,15 / 1,20 / 1$ \\
\hline
\end{tabular}

La licuefacción hidrotérmica de biomasa se llevó a cabo en un reactor PARR4576 B de $250 \mathrm{~mL}$, el cual fue cargado con la biomasa, el solvente y el catalizador. Posteriormente, se presurizó con argón (Ar) hasta una presión de 10 bares y se inició el calentamiento hasta la temperatura deseada con una agitación de 360 rpm, una vez alcanzada la temperatura de reacción se empezó a contabilizar el tiempo de reacción; después del cual, el reactor se dejó enfriar hasta temperatura ambiente y se procedió a la separación de los productos de reacción.

\section{Separación de productos de reacción}

Después de enfriar el reactor, se procedió a realizar la separación de productos y subproductos de reacción. Los productos gaseosos de la reacción de licuefacción se retiraron del reactor al alcanzar la temperatura ambiente. La mezcla de reacción se separó mediante filtración al vacío en una fracción sólida y una fracción líquida. La fracción sólida se lavó luego con acetona suficiente para extraer el biocrudo. Esta mezcla de acetona biocrudo se separó por evaporación al vacío a $40^{\circ} \mathrm{C}$ para recuperar la acetona y obtener biocrudo. El biocrudo contenido en la fracción líquida se extrajo con diclorometano. La mezcla diclorometano biocrudo se separó posteriormente con evaporación al vacío a $35^{\circ} \mathrm{C}$ para recuperar diclorometano y obtener biocrudo soluble en agua (Oasmaa et al., 2003; Garcia-Perez et al., 2007; Yang et al., 2014; Zou et al., 2009). Los biocrudos resultantes se mezclaron y el total fue cuantificado y reportado como rendimiento de biocrudo. La Fig. 1 muestra el procedimiento empleado en la separación de productos de reacción. Como puede observarse, luego de extraer los solventes mediante evaporación, los productos de interés de la reacción de licuefacción fueron biocrudo total, residuo sólido (RS) y fase acuosa (FA), los cuales fueron almacenados para su posterior cuantificación y caracterización.

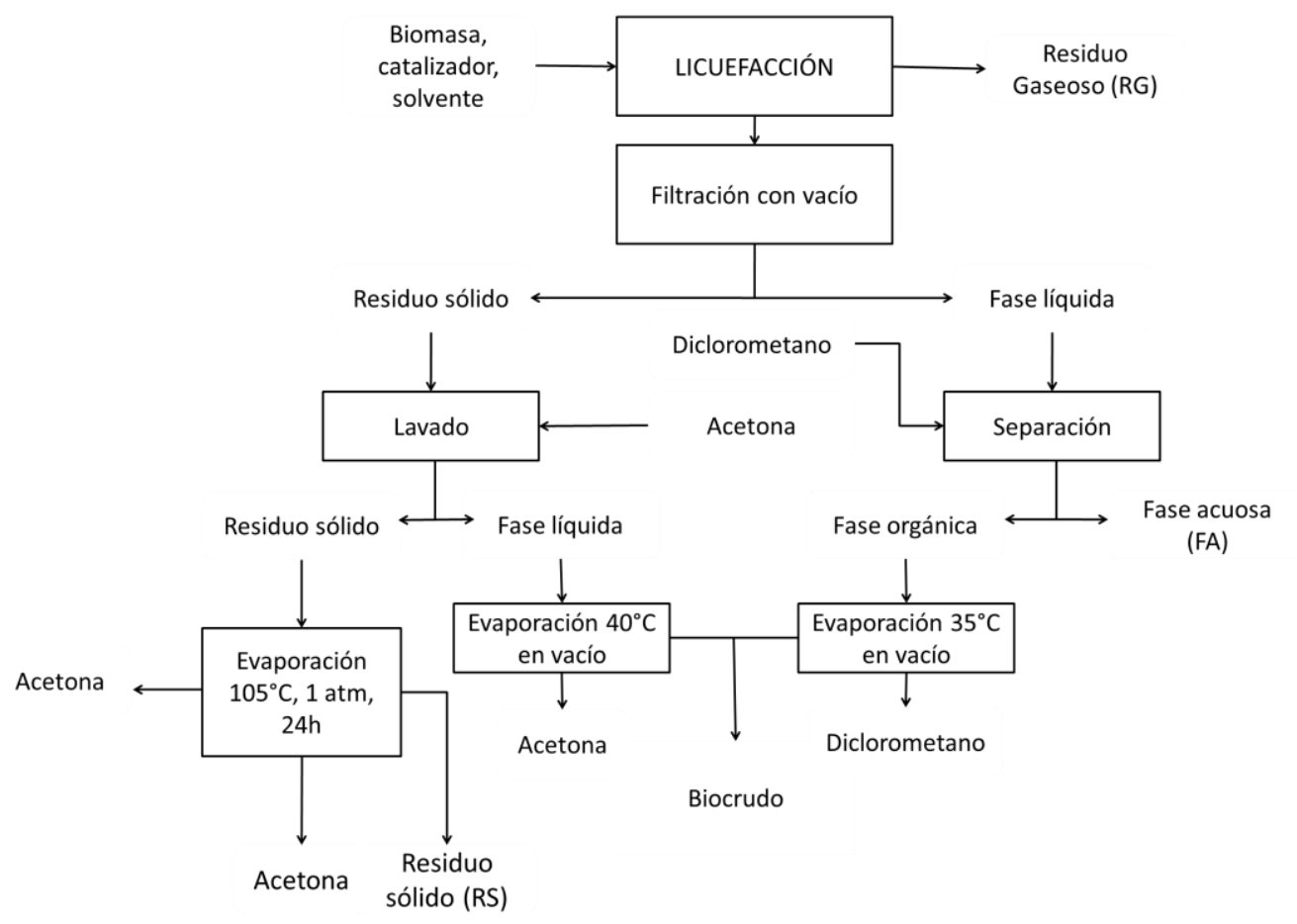

Fig. 1: Procedimiento para la separación de productos y sub-productos de reacción

Para calcular los rendimientos hacia las diferentes fracciones se emplearon las siguientes ecuaciones, teniendo en cuenta el contenido de materia orgánica presente en la biomasa inicialmente alimentada. Para estas ecuaciones se empleó el peso de los productos y subproductos secos. 


$$
\begin{aligned}
& \% \text { Rendimiento Biocrudo }=\frac{\text { peso Biocrudo }}{\text { peso biomasa inicial }} \times 100 \\
& \% \text { Rendimiento Residuo Sólido }=\frac{\text { peso Residuo Sólido }}{\text { peso biomasa inicial }} X 100 \\
& \% \text { Rendimiento Residuo Acuoso }=\frac{\text { peso Residuo Acuoso }}{\text { peso biomasa inicial }} X 100
\end{aligned}
$$

Rendimiento Energético $(R E)=\%$ Rendimiento Biocrudo X HHV

\section{Caracterización de productos}

El biocrudo obtenido después de la reacción fue caracterizado mediante FTIR (Thermo Nicolet 6700), empleando la celda de ATR para determinar los principales grupos funcionales. Las muestras de biocrudo fueron disueltas en diclorometano y analizadas cromatografía gaseosa acoplada a masas GC-MS en un cromatógrafo Agilent Technology 7890A acoplado a un detector de masas 5975C. Se utilizó una columna capilar no polar DB-5HT (30 m, $0.25 \mathrm{~mm}$ y $0.10 \mu \mathrm{m})$ para separar los componentes y se empleó como estándar interno tetradecano para cuantificar la proporción de los principales componentes del biocrudo. Las composiciones elementales del biocrudo fueron determinadas usando un analizador elemental TruSepc CHNS-O micro marca Leco. El poder calorífico superior del biocrudo fue determinado usando la fórmula de Dulong (Duan y Savage, 2011; Duan et al., 2013; Yuan et al., 2009; Zhuang et al., 2012).

$$
H H V(M J / k g)=0.338 C+1.428(H-O / 8)
$$

Donde $\mathrm{C}, \mathrm{H}, \mathrm{O}$, corresponden a las composiciones de cada elemento en el material.

\section{RESULTADOS Y DISCUSIÓN}

Los resultados se presentan en las secciones: Preparación de la muestra, evaluación de las condiciones del proceso de licuefacción hidrotérmica de biomasa y caracterización del biocrudo.

\section{Preparación de la muestra}

Las muestras de Buchón de Agua se homogenizaron mediante reducción del tamaño de partícula en un molino de cuchillas (833-1397 $\mu \mathrm{m})$. Se analizó la composición elemental del Buchón de Agua, se calculó el poder calorífico HHV y se determinó el contenido de cenizas a $575^{\circ} \mathrm{C}$ (ver Tabla 2)

Tabla 2: Caracterización biomasa Buchón de Agua en base seca

\begin{tabular}{ll}
\hline Carbono, \% & 29,21 \\
Hidrógeno, \% & 4,82 \\
Oxígeno (por diferencia), \% & 63,69 \\
Nitrógeno, \% & 2,26 \\
Azufre, \% & 0,01 \\
HHV (fórmula de Dulong), MJ/Kg & 11,43 \\
Cenizas, \% & 9,44 \\
\hline
\end{tabular}

\section{Evaluación de condiciones del proceso de licuefacción hidrotérmica de biomasa}

Con el fin de determinar el efecto de variables como el tiempo de reacción, la temperatura y la relación líquido/sólido en el proceso de licuefacción hidrotérmica de Buchón de Agua, se desarrolló el diseño experimental mostrado en la Tabla 3. Para efectos de comparación, se realizó un experimento de licuefacción sin catalizador (Blanco) a $270^{\circ} \mathrm{C}, 30$ min y con una relación líquido/sólido de 20.

Tabla 3: Diseño Experimental licuefacción hidrotérmica de biomasa

\begin{tabular}{cccc}
\hline Experimento & Temperatura $\left({ }^{\circ} \mathrm{C}\right)$ & Relación líquido/sólido $(\mathrm{g} / \mathrm{g})$ & Tiempo (min) \\
\hline 1 & 300 & 15 & 0 \\
2 & 270 & 10 & 0 \\
3 & 330 & 10 & 0 \\
4 & 330 & 15 & 30 \\
\hline
\end{tabular}


Tabla 3 (continuación)

\begin{tabular}{|c|c|c|c|}
\hline 5 & 300 & 10 & 30 \\
\hline 6 & 330 & 15 & 0 \\
\hline 7 & 270 & 10 & 30 \\
\hline 8 & 330 & 20 & 60 \\
\hline 9 & 270 & 20 & 60 \\
\hline 10 & 330 & 20 & 0 \\
\hline 11 & 300 & 20 & 60 \\
\hline 12 & 300 & 15 & 30 \\
\hline 13 & 270 & 10 & 60 \\
\hline 14 & 300 & 20 & 0 \\
\hline 15 & 300 & 10 & 60 \\
\hline 16 & 270 & 15 & 30 \\
\hline 17 & 330 & 10 & 30 \\
\hline 18 & 330 & 15 & 60 \\
\hline 19 & 300 & 15 & 60 \\
\hline 20 & 270 & 15 & 0 \\
\hline 21 & 270 & 15 & 60 \\
\hline 22 & 300 & 20 & 30 \\
\hline 23 & 330 & 20 & 30 \\
\hline 24 & 330 & 10 & 60 \\
\hline 25 & 270 & 20 & 0 \\
\hline 26 & 270 & 20 & 30 \\
\hline 27 & 300 & 10 & 0 \\
\hline Blanco & 270 & 20 & 30 \\
\hline
\end{tabular}

La Fig. 2, muestra la distribución de productos obtenidos durante la licuefacción hidrotérmica de Buchón de Agua a 270,300 y $330^{\circ} \mathrm{C}$ en base seca y libre de inorgánicos. Como puede observarse, a $270^{\circ} \mathrm{C}$ la cantidad de residuo acuoso aumenta de acuerdo al aumento en la relación de líquido/sólido utilizada. Para los casos en los que se llevaron a cabo las reacciones a $300^{\circ} \mathrm{C}$ y $330^{\circ} \mathrm{C}$, la cantidad de residuo acuoso es mayor en relaciones líquido/sólido de $15 \mathrm{~g} / \mathrm{g}$, indicando que a altas temperaturas el agua reacciona más eficientemente con la biomasa para formas biocrudo. En general, la conversión de la biomasa aumenta al aumentar la temperatura. El rendimiento a biocrudo no muestra una correlación clara con las condiciones de reacción. A $270^{\circ} \mathrm{C}$, puede apreciarse que el rendimiento tiende a aumentar con el tiempo de reacción, pero disminuye con el aumento en la cantidad de solvente empleada. Por otro lado, el mayor rendimiento a biocrudo se obtuvo bajo condiciones de $300^{\circ} \mathrm{C}, 30$ minutos y una relación líquido/sólido de 10.

De acuerdo al análisis estadístico realizado (que no se muestra en este paper), no fue posible establecer una relación directa entre las variables experimentales con el rendimiento de biocrudo, para la biomasa del Buchón de Agua. Lo cual sugiere que podría ser favorable la disminución tanto en la temperatura como en el tiempo de reacción; sin embargo, se debe considerar el poder calorífico del biocrudo obtenido, como se mostrará más adelante de acuerdo a la composición elemental obtenida para cada uno de los experimentos realizados. Es importante además tener en cuenta que, debido a la estructura y composición fisicoquímica del Buchón de Agua, las condiciones evaluadas favorecen la descomposición tanto de la celulosa, hemicelulosa y lignina, como de los compuestos derivados de la degradación térmica de estos, además de otros compuestos que conforman la biomasa como las grasas, ceras, colorantes, entre otros. Es importante considerar, que, de los productos y subproductos de la reacción de licuefacción, es indeseable obtener un alto rendimiento de formación de gases, ya que esto da cuenta de una pérdida en la eficiencia de la reacción, además los compuestos que forman ésta fase son menos valiosos que los compuestos que forman la fase acuosa y sólida. 


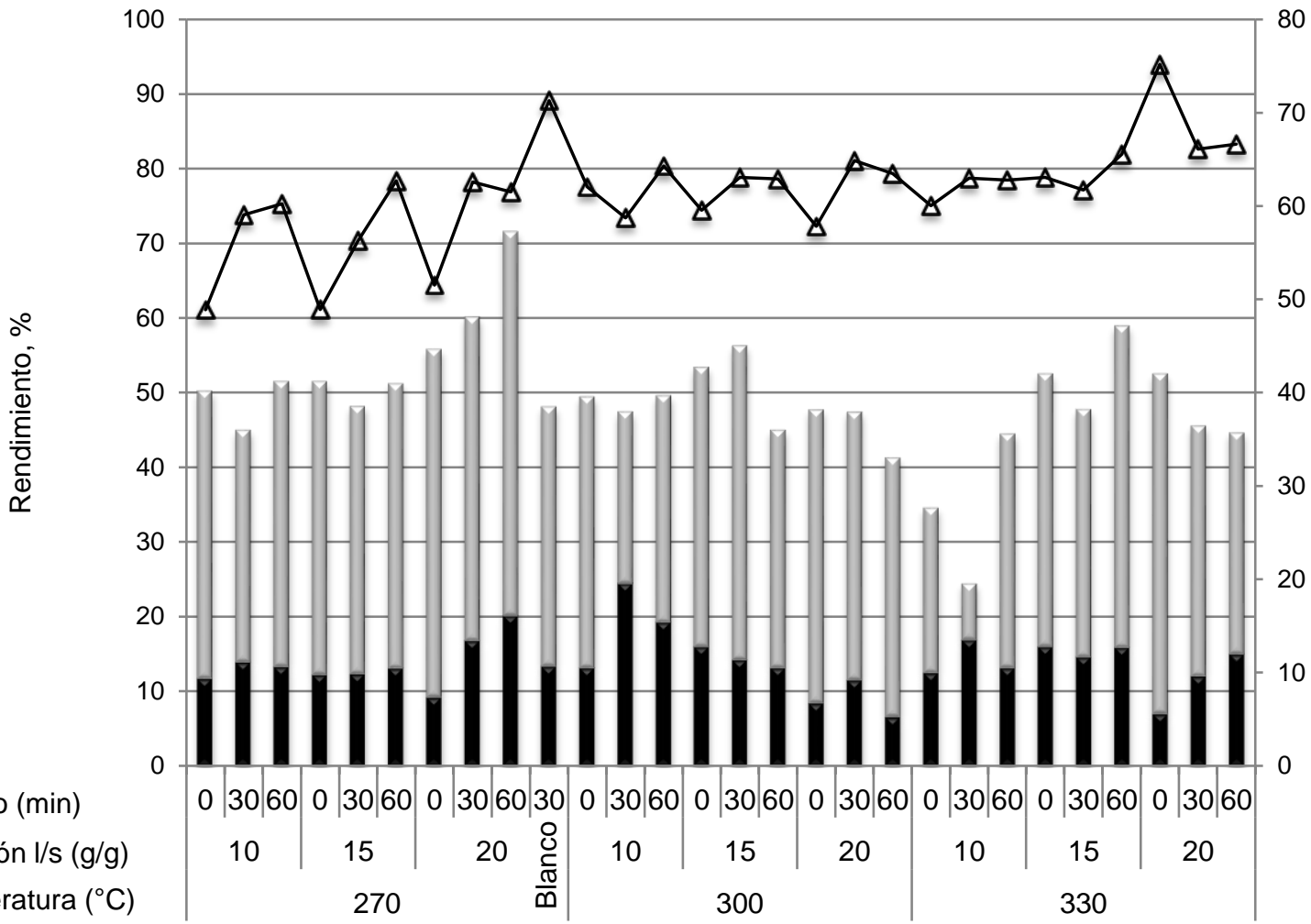

Tiempo (min)

Relación $1 / \mathrm{s}(\mathrm{g} / \mathrm{g})$

Temperatura $\left({ }^{\circ} \mathrm{C}\right)$

270

$\triangle$ Conversión de Biomasa (CB)

Fig. 2: Rendimientos de Biocrudo, Residuo Sólido (RS) y Residuo Acuoso (RA).

En la Tabla 4, se presentan los valores obtenidos para el poder calorífico así como la composición elemental de la biomasa y las muestras de biocrudo obtenidas durante el desarrollo experimental. En general se observa un aumento significativo en la densidad energética del biocrudo obtenido en comparación con la biomasa cruda. Asimismo, es evidente el aumento del poder calorífico del biocrudo en los experimentos del diseño en comparación con el poder calorífico del biocrudo obtenido en el blanco, lo que indica la gran influencia del catalizador en el proceso de licuefacción hidrotérmica de la biomasa, fenómeno observado más directamente en los análisis elementales, en los que puede verse el aumento significativo del contenido de carbono y la disminución del contenido de oxígeno en los biocrudos del diseño, en comparación con los resultados del análisis elemental del blanco. El aumento en el poder calorífico logrado bajo las diferentes condiciones de reacción empleadas, implica polimerizaciones secundarias, generadas por reacciones de oxidación ocurridas entre los productos primarios de hidrólisis de los componentes. Este aumento en la densidad energética, es un aspecto de gran importancia a la hora de considerar la factibilidad económica de este tipo de procesos de densificación energética de biomasas como las estudiadas hasta ahora en este proyecto.

Tabla 4: Poder calorífico y composición elemental del biocrudo en base seca

\begin{tabular}{cccccc}
\hline Experimento & $N$ & $C$ & $H$ & $O$ & $H H V(M J / k g)$ fórmula de Dulong \\
\hline Buchón de Agua & 2,26 & 29,21 & 4,82 & 63,69 & 11,43 \\
\hline 1 & 3,03 & 79,56 & 8,36 & 10,13 & 37,00 \\
2 & 3,89 & 79,89 & 7,58 & 12,94 & 35,50 \\
3 & 4,27 & 78,99 & 6,99 & 10,26 & 34,84 \\
4 & 4,14 & 77,53 & 6,76 & 13,46 & 33,44 \\
5 & 4,23 & 57,49 & 6,48 & 15,92 & 25,83 \\
6 & 3,32 & 71,98 & 7,55 & 13,74 & 32,65 \\
7 & 3,90 & 69,26 & 6,64 & 13,94 & 30,40 \\
8 & 3,60 & 78,43 & 6,51 & 14,31 & 33,25 \\
9 & 4,23 & 71,07 & 6,93 & 14,85 & 31,26 \\
10 & 2,82 & 61,76 & 6,50 & 13,12 & 27,80 \\
\hline
\end{tabular}


Tabla 4 (continuación)

\begin{tabular}{cccccc}
\hline 11 & 3,79 & 55,10 & 6,72 & 14,02 & 25,71 \\
12 & 3,72 & 76,83 & 7,42 & 11,95 & 34,42 \\
13 & 4,62 & 73,92 & 7,23 & 15,07 & 32,61 \\
14 & 5,75 & 67,98 & 6,80 & 14,66 & 32,06 \\
15 & 4,86 & 74,32 & 7,20 & 13,78 & 32,35 \\
16 & 5,77 & 72,88 & 7,09 & 13,46 & 34,72 \\
17 & 6,35 & 76,06 & 7,90 & 12,64 & 31,72 \\
18 & 5,38 & 71,44 & 7,30 & 15,92 & 26,82 \\
19 & 4,57 & 61,20 & 6,25 & 15,59 & 31,16 \\
20 & 5,35 & 69,52 & 7,35 & 15,82 & 33,15 \\
21 & 5,78 & 72,68 & 7,63 & 12,89 & 34,14 \\
22 & 4,30 & 74,14 & 8,02 & 13,20 & 34,37 \\
23 & 7,03 & 76,36 & 7,55 & 12,35 & 34,81 \\
24 & 6,28 & 77,11 & 7,58 & 11,53 & 32,61 \\
25 & 6,32 & 71,52 & 7,36 & 11,50 & 34,92 \\
26 & 4,85 & 74,60 & 8,68 & 14,99 & 32,24 \\
27 & 6,99 & 73,82 & 7,16 & 16,41 & 24,68 \\
Blanco & 4,23 & 56,21 & 5,97 & 15,92 & \\
\hline
\end{tabular}

Se puede observar en general para las muestras de biocrudo obtenidas, un aumento en el contenido de carbono y una disminución significativa en el contenido de oxígeno con respecto a la materia prima inicial. El aumento en el contenido de hidrógeno es especialmente significativo para las muestras de biocrudo obtenido de Buchón de Agua. La Fig. 3 muestra los resultados de Poder calorífico (HHV) y Rendimiento de Biocrudo. Los resultados se muestran de menor a mayor temperatura de reacción $\left(270^{\circ} \mathrm{C}, 300^{\circ} \mathrm{C}\right.$ y $\left.330^{\circ} \mathrm{C}\right)$, menor a mayor relación líquido/sólido $(10 \mathrm{~g} / \mathrm{g}, 15 \mathrm{~g} / \mathrm{g}$ y $20 \mathrm{~g} / \mathrm{g})$ y de menor a mayor tiempo de reacción $(0 \mathrm{~min}, 30 \mathrm{~min}$ y $60 \mathrm{~min})$. En los resultados sobresale el mayor poder calorífico $\left(37 \mathrm{MJ} / \mathrm{Kg}\right.$ ) obtenido a $300^{\circ} \mathrm{C}, 15 \mathrm{~g} / \mathrm{g}$ y 0 min de tiempo de reacción y el mayor rendimiento de biocrudo $(24,4 \%)$ obtenido a $300^{\circ} \mathrm{C}, 10 \mathrm{~g} / \mathrm{g}$ y $30 \mathrm{~min}$ de reacción. En la gráfica general de resultados experimentales puede intuirse que no hay una relación directa entre las variables de salida (poder calorífico y rendimiento total de biocrudo) y las variables de entrada del diseño experimental (temperatura, tiempo de reacción y relación líquido/sólido).

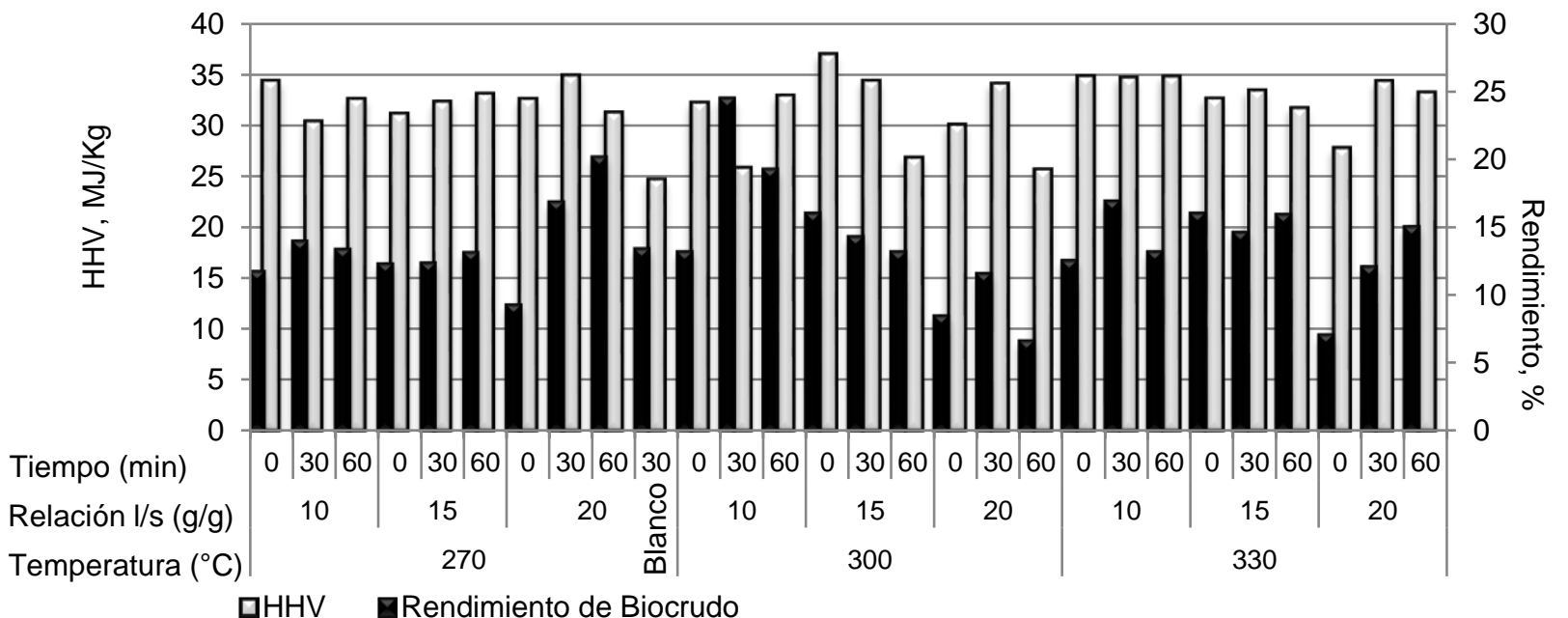

Fig. 3: Rendimiento a biocrudo y poder calorífico para la licuefacción hidrotérmica de Buchón de Agua.

Debido a que los valores de rendimiento a biocrudo y su poder calorífico no siguen una misma tendencia y esto dificulta la selección de las mejores condiciones de proceso, se propuso la variable respuesta denominada Rendimiento Energético (RE), que es la cantidad de energía contenida en el biocrudo que se obtiene por cada $1 \mathrm{Kg}$ de biomasa alimentada; ésta se obtiene multiplicando el rendimiento a biocrudo por su 
poder calorífico y dividiendo por 100. La Fig. 4 muestra la variación decreciente de RE en función del número del experimento, el cual a su vez se puede correlacionar con las condiciones experimentales de la Tabla 3.

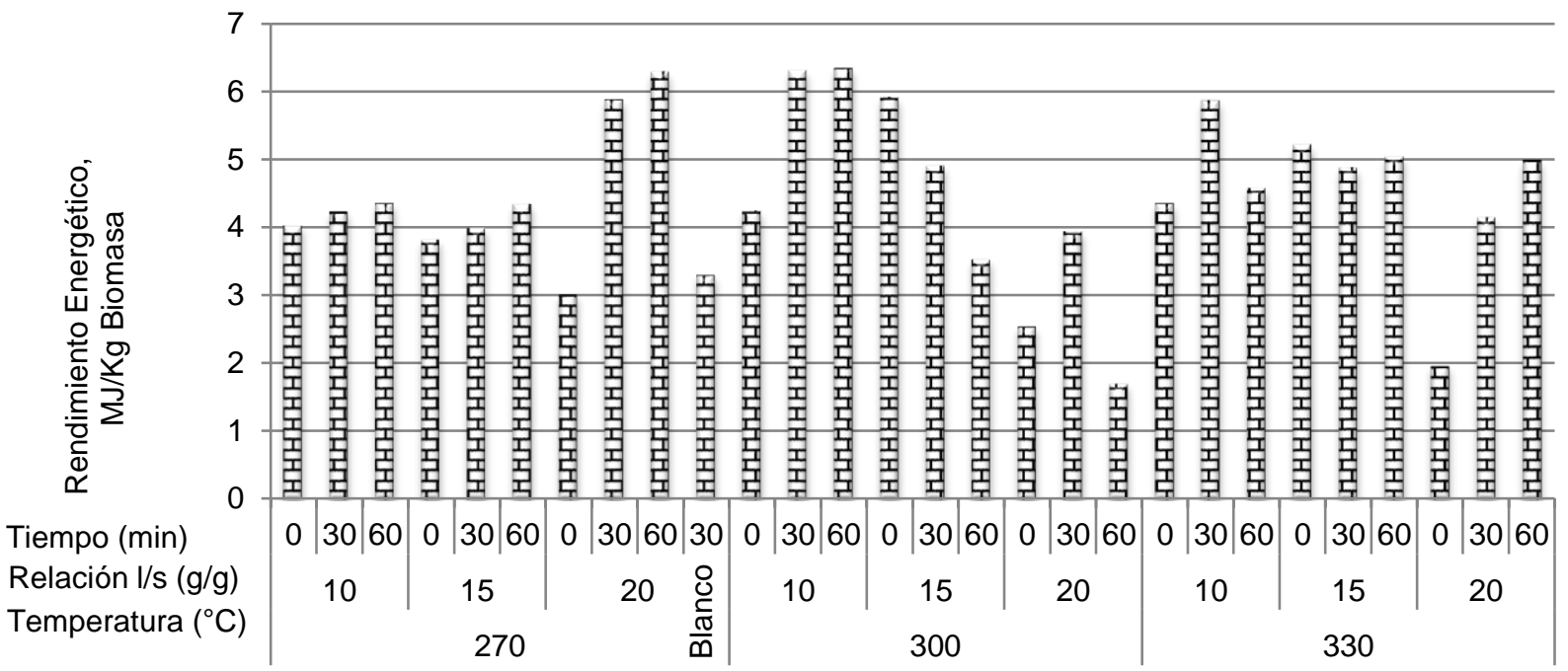

Fig. 4: Rendimiento Energético (RE) del biocrudo según las condiciones experimentales.

Los experimentos con los mayores rendimientos energéticos y sus respectivas condiciones de operación son: experimento $15\left(300^{\circ} \mathrm{C}, 60\right.$ min y $\left.\mathrm{L} / \mathrm{S}=10\right)$ con Rendimiento Energético de $6,34 \mathrm{MJ} / \mathrm{Kg}$ biomasa, experimento $5\left(300^{\circ} \mathrm{C}, 30 \mathrm{~min}\right.$ y L/S=10) con $\mathrm{RE}$ de $6,31 \mathrm{MJ} / \mathrm{Kg}$ biomasa y experimento $9\left(270^{\circ} \mathrm{C}, 60 \mathrm{~min}\right.$ y $\left.\mathrm{L} / \mathrm{S}=20\right)$ con $6,29 \mathrm{MJ} / \mathrm{Kg}$ biomasa. Considerando su menor relación L/S y el menor tiempo de reacción, se puede establecer que el mejor resultado se obtuvo con las condiciones del experimento 5 . Al comprar el experimento $26\left(270^{\circ} \mathrm{C}\right.$, $30 \mathrm{~min}$ y $20 \mathrm{~g} / \mathrm{g}$ ) y el Blanco, que se realizaron bajo las mismas condiciones excepto que el blanco no se adicionó catalizador, es evidente que tanto el rendimiento a biocrudo como su poder calorífico son mayores cuando se adiciona catalizador. La ventaja de adicionar catalizador se hace más evidente cuando se comparan los resultados del RE del experimento con catalizador (5,9 MJ/Kg biomasa) y del experimento sin catalizador (3,3 MJ/Kg biomasa).

\section{Análisis estadístico}

Para el diseño experimental se usó un modelo cúbico con el fin de lograr un mejor ajuste, ya que modelos cuadráticos arrojaban ajustes en las correlaciones por debajo de $50 \%$. El análisis ANOVA de la variable respuesta Rendimiento a Biocrudo mostró que el modelo propuesto es significante ( $p$-value de 0,0191 ), con solo a variable Relación líquido-sólido dentro de sus términos significantes ( $p$-value de 0,0005 ). Asimismo, el coeficiente de correlación $\left(\mathrm{R}^{2}\right)$ arrojado por el análisis, 0,7625, es aceptable. El análisis ANOVA de la variable respuesta Poder Calorífico (HHV) mostró que el modelo propuesto no es estadisticamente significante ( $p$ value de 0,1355 ), con solo a variable Tiempo dentro de sus términos significantes ( $p$-value de 0,0183 ). Asimismo, el coeficiente de correlación $\left(R^{2}\right)$ arrojado por el análisis, 0,6551 , indica que el ajuste del modelo es bajo y por tanto no describe los resultados apropiadamente. En cuando a la variable respuesta Rendimiento Energético, el coeficiente de correlación $\left(R^{2}\right)$ del modelo fue de 0,7730, que es el mayor valor obtenido para todas las variables respuesta investigadas. El p-value del modelo fue de 0,015 , que confirma el buen ajuste del modelo. Las variables Tiempo y Relación Líquido/sólido tuvieron p-values de 0,05 y 0,0063, respectivamente, por lo cual se considera que tienen efecto estadisticamente significativos sobre la respuesta. La temperara no tuvo efecto significativo pues su p-value fue de 0,1893. Los resultados del análisis ANOVA se condensan en la Tabla 5.

Tabla 5: Valores p modelo propuesto para las variables Rendimiento de Biocrudo, Poder Calorífico y Rendimiento Energético

\begin{tabular}{|c|c|c|c|}
\hline Factor & Rendimiento Biocrudo & Poder Calorífico & Rendimiento Energético \\
\hline Modelo & 0,0194 & 0,1355 & 0,0150 \\
\hline A-Temperatura & 0,2521 & 0,5558 & 0,1893 \\
\hline B-Tiempo & 0,2918 & 0,0183 & 0,0536 \\
\hline C-Relación líquido-sólido & 0,0005 & 0,0713 & 0,0063 \\
\hline$A B$ & 0,5816 & 0,4945 & 0,7062 \\
\hline$A C$ & 0,0871 & 0,2191 & 0,0357 \\
\hline $\mathrm{BC}$ & 0,3399 & 0,9154 & 0,3140 \\
\hline$A^{2}$ & 0,9537 & 0,2000 & 0,6394 \\
\hline
\end{tabular}


Tabla 5 (continuación)

\begin{tabular}{llll}
\hline $\mathrm{B}^{2}$ & 0,0837 & 0,1266 & 0,0428 \\
$\mathrm{C}^{2}$ & 0,8550 & 0,2279 & 0,4443 \\
$\mathrm{ABC}$ & 0,7885 & 0,4553 & 0,9504 \\
$\mathrm{~A}^{2} \mathrm{~B}$ & 0,2130 & 0,0421 & 0,0625 \\
$\mathrm{~A}^{2} \mathrm{C}$ & 0,0012 & 0,7057 & 0,0035 \\
$\mathrm{AB}^{2}$ & 0,9257 & 0,4914 & 0,7861 \\
$\mathrm{AC} 2$ & 0,0985 & 0,9275 & 0,1161 \\
$\mathrm{~B}^{2} \mathrm{C}$ & 0,3995 & 0,0048 & 0,5992 \\
$\mathrm{BC}^{2}$ & 0,0649 & 0,2453 & 0,0206 \\
\hline
\end{tabular}

La Fig. 5 muestra los efectos de las diferentes variables de entrada (Temperatura, Tiempo de reacción y Relación liquido-sólido) sobre el Rendimiento Energético, que da cuenta de los cambios en poder calorífico y rendimiento de biocrudo en conjunto. Se observa el efecto más marcado de las variables tiempo y relación líquido-solido. Cambios en el tiempo de reacción de 60 min significan, según el diagrama, un aumento de 0,70 $\mathrm{MJ} / \mathrm{Kg}$ de biomasa, con un máximo de $5 \mathrm{MJ} / \mathrm{Kg}$ de biomasa alrededor de los 40 min de reacción, y un aumento de 10 en la relación líquido-solido una disminución de 1,10 MJ/Kg de biomasa, con un máximo de 5,30 MJ/100 $\mathrm{Kg}$ biomasa con una relación de $10 \mathrm{~g} / \mathrm{g}$. La variable temperatura, por su parte, tiene un efecto muy leve sobre el rendimiento energético, con una tasa de cambio de $0,15 \mathrm{MJ} / \mathrm{Kg}$ de biomasa por cada $60^{\circ} \mathrm{C}$ de cambio en la temperatura de reacción.

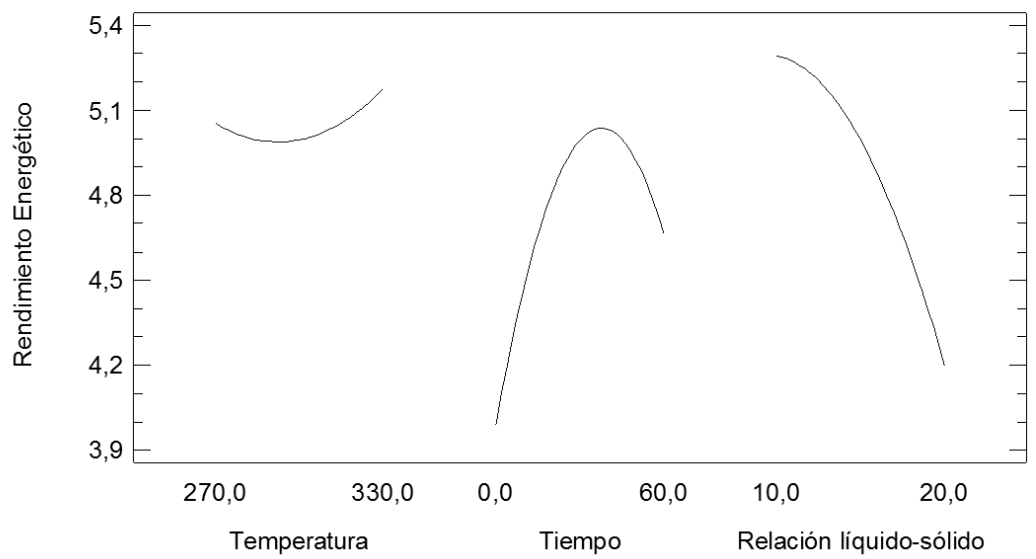

Fig. 5: Efectos de temperatura, tiempo y relación líquido-sólido sobre el Rendimiento Energético.

\section{Caracterización adicional del biocrudo}

Las fracciones de biocrudo producidas durante la licuefacción hidrotérmica de Buchón de Agua se caracterizaron mediante cromatografía gaseosa acoplada a un detector de masas, con el fin de determinar el tipo de compuestos que conforman esta fase y mediante análisis de infrarrojo con transformada de Fourier para determinar los grupos funcionales presentes.

\section{GC-Masas del Biocrudo}

Los resultados mostraron una mayor formación de compuestos para las mayores relaciones líquido-sólido y tiempo de reacción, debido principalmente a la degradación de las unidades estructurales de la lignina. Se evidencia una proporción importante de compuestos de bajo peso molecular como esteres, furanos y compuestos aromáticos, como fenol, 2- metoxi fenol, vinil furano, 2,6- metoxi fenol. Se observa además la presencia de ácidos grasos como ácido oleico y ácido hexadecanoico, compuestos nitrogenados como pirroles, hidrocarburos de cadena larga como tetracosano y en algunos casos la presencia en una proporción bastante considerable de compuestos derivados de la degradación del material plástico contenido en las muestras de biomasa sometidas a reacción.

En general, la formación de los diferentes compuestos evidenciados en todas las muestras de biocrudo obtenidas durante la licuefacción de Buchón de Agua se debe a la combinación de las variables involucradas en la reacción, no sólo las estudiadas hasta este momento (temperatura, tiempo y relación biomasa: solvente) si no también el tipo y carga de catalizador, el tamaño de partícula, el tipo de solvente, etc. Los resultados cromatográficos obtenidos para las tres biomasas, evidencian compuestos de degradación de la lignina como 2- metoxi-fenol; de acuerdo con mecanismos propuestos para la degradación de ésta fracción vía especies de radicales libres (Karagoz et al., 2005), los grupos hidroxilos alquílicos en la posición a son eliminados primero de la cadena lateral de propano seguido por el rompimiento de un metil-éter formando monolignoles, 
principalmente alcohol cumarílico, alcohol sinapílico y alcohol coniferílico (ver esquema de la 3). La transferencia de hidrógeno del grupo hidroxilo en la posición $\gamma$, a especies de radicales libres lleva a la formación de metoxi fenoles, dímeros y formaldehido.

Compuestos como las cetonas presentes en todas las muestras, se derivan principalmente de la degradación de carbohidratos y los compuestos nitrogenados como aminas y amidas se derivan de la degradación del contenido proteico en el caso del Buchón de Agua. Por otra parte, debido a la presencia de clorofila como principal colorante de la biomasa acuática, también se observó en algunas muestras la presencia de fitol, producido por la hidrólisis de la molécula de clorofila (Duan et al., 2011). Los resultados obtenidos en el análisis cromatográfico muestran concordancia con el abanico de compuestos presentes en todo el mecanismo propuesto para la licuefacción hidrotérmica de la biomasa, donde proteínas, carbohidratos, lignina y lípidos se rompen primero dando como resultado sus correspondientes monómeros tales como aminoácidos, glucosa, xilosa, fenoles y ácidos grasos. Estos monómeros se descomponen a su vez en varios tipos de intermediarios, donde posteriormente los monómeros y sus intermediarios sufren una serie de reacciones durante el proceso para formar el producto sólido, líquido y gaseoso (Zhang et al., 2013b).

\section{FTIR del biocrudo}

La fracción orgánica insoluble en agua o biocrudo, fue analizada mediante espectroscopía FTIR, para identificar los principales grupos funcionales de los compuestos que forman esta mezcla compleja. En la Tabla 5 se muestran las principales bandas para el análisis del biocrudo obtenido en la licuefacción de las tres biomasas.

Tabla 6: Grupos funcionales identificados mediante FTIR en el biocrudo

\begin{tabular}{lll}
\hline $\mathrm{cm}^{-1}$ & Grupo funcional & Tipo de compuesto \\
\hline $3600-3300$ & Estiramiento O-H, N-H & O-H poliméricos, agua \\
$3500-2800$ & Estiramiento C-H & Grupos metil y metileno de alcanos y ácidos grasos \\
$1750-1650$ & Estiramiento $\mathrm{C}=\mathrm{O}$ & Cetonas, aldehídos, ácido carboxílicos \\
$1680-1580$ & Estiramiento $\mathrm{C}=\mathrm{C}$ & Alquenos \\
$1470-1350$ & Estiramiento $\mathrm{C}-\mathrm{H}$ & Alcanos, compuestos alquílicos sustituidos \\
$1300-950$ & Estiramiento C-O & Alcoholes primarios, secundarios y terciarios \\
$1250-1020$ & Estiramiento C-N & Aminas aromáticas \\
$915-650$ & Deformación O-H & Fenoles, esteres, éteres y compuestos aromáticos mono cíclicos, \\
& & policíclicos y sustituidos. \\
\hline
\end{tabular}

Los resultados del análisis FTIR reflejan principalmente la formación de numerosos compuestos provenientes de la degradación de la fracción lignocelulósica del material, así como también de otros compuestos como ceras, proteínas, colorantes, grasas, etc. En los espectros FTIR obtenidos para todas las muestras (no mostrados), se pudo observar esencialmente la existencia de compuestos aromáticos, ácidos grasos, hidrocarburos alifáticos, cetonas, aldehídos y alcoholes. Además, se obtuvo, que, con el aumento en las condiciones de reacción, aumenta la intensidad de las bandas exhibidas en el rango $800-4000 \mathrm{~cm}^{-1}$, principalmente las bandas asociadas a la formación de compuestos como alcoholes, ácidos carboxílicos, cetonas y aromáticos. La banda entre $3300-3600 \mathrm{~cm}^{-1}$, puede deberse además a la presencia de compuestos derivados de la degradación y reacción de la fracción proteica del material vegetal, por lo cual pudieron observarse picos entre 1020-1130 $\mathrm{cm}^{-1}$. Además, al comparar los espectros obtenidos, se puede apreciar que la intensidad en las bandas de absorción entre 2850-2960 $\mathrm{cm}^{-1}$ aumenta con la disminución en la relación líquido/sólido. Para las tres condiciones de temperatura evaluadas y con una relación líquido/sólido de 15 a un tiempo de 30 minutos, se pudo observar un aumento en las bandas alrededor de $1280 \mathrm{~cm}^{-1}$ debido a la presencia de alcoholes y cetonas.

\section{Caracterización del Residuo Sólido (RS) y de la Fracción Acuosa (FA)}

Tanto el subproducto sólido como el líquido acuoso, han sido caracterizados mediante FTIR, para identificar algunos grupos funcionales de los compuestos formados durante la licuefacción hidrotérmica, como producto de la degradación sucesiva de la celulosa, hemicelulosa y lignina que conforman las diferentes biomasas.

\section{FTIR del Residuo Sólido (RS)}

Durante el análisis de los espectros obtenidos para los residuos sólidos de la reacción, se observaron diferentes bandas características, cuya interpretación se muestra en la Tabla 6. En el residuo sólido del Buchón de Agua aún puede observarse picos característicos de celulosa, hemicelulosa y lignina, que indican una conversión incompleta de la biomasa bajo las diferentes condiciones de reacción evaluadas. 
La conversión de la biomasa aumentó con el tiempo de reacción y con la cantidad de solvente empleado para una temperatura de $270 \stackrel{\circ}{ } \mathrm{C}$; para las demás condiciones experimentales, se observó un comportamiento diferente, el cual es asociado a la heterogeneidad de la materia prima en cuestión, la cual presenta una proporción considerable de compuestos externos a la biomasa como plásticos, los cuales son más difíciles de degradar bajo las condiciones de reacción evaluadas y por lo tanto tienen una presencia significativa en el residuo sólido resultante de la licuefacción hidrotérmica del Buchón de Agua, lo cual también es evidenciado por la intensidad de las bandas entre $3000-2800 \mathrm{~cm}^{-1}$ relacionados con la presencia de estructuras alifáticas resistentes al calor. El residuo sólido formado, también incluye compuestos re polimerizados en la fase acuosa o en la fase biocrudo. Por otra parte, los cambios en las bandas 1501, 1425 y 1060, evidencian la licuefacción principalmente de la celulosa y hemicelulosa, ya que la mayoría de las bandas exhibidas por los espectros IR muestran la presencia de grupos funcionales correspondientes principalmente a las diferentes fracciones de la lignina. Al parecer el aumento en la cantidad de solvente empleado para las reacciones de licuefacción favorece la degradación de la biomasa, disminuyendo así la cantidad de residuo sólido.

Tabla 7: Grupos funcionales identificados mediante FTIR en el residuo sólido

\begin{tabular}{cl}
\hline $\mathrm{cm}^{-1}$ & \\
\hline 3400 & Estiramiento $\mathrm{O}-\mathrm{H}$ \\
2920 & Estiramiento de $\mathrm{CH}$ y $\mathrm{CH}_{2}$ de unidades guayacilo de la lignina \\
2850 & Estiramiento $\mathrm{C}-\mathrm{H}$ de metil y metileno \\
1739 & Estiramiento $\mathrm{C}=\mathrm{O}$ de la hemicelulosa \\
$1633-1500$ & Enlace $-\mathrm{C}=\mathrm{C}-$ del anillo aromático de la unidad p-hidroxifenil propano de la lignina \\
$1463-1425$ & Grupo metoxi o- $\mathrm{CH}_{3}$, deformación C-H de la lignina \\
1373 & Flexión $-\mathrm{C}-\mathrm{H}-$ de celulosa, hemicelulosa y lignina \\
1330 & Vibración C-H, flexión O-H de celulosa, hemicelulosa y lignina \\
1240 & Deformación C-O-H y estiramiento C-O en fenoles \\
1200 & Flexión O-H de celulosa y hemicelulosa \\
1160 & Estiramiento asimétrico de C-O-C de celulosa y hemicelulosa \\
1100 & Vibración C-O de celulosa cristalina, estiramiento de la glucosa de la celulosa \\
$1060-1035$ & Vibración C-O de celulosa \\
900 & Vibración C-O de celulosa amorfa \\
\hline
\end{tabular}

\section{FTIR de la Fracción Acuosa (FA)}

Durante la licuefacción de la biomasa de Buchón de Agua se evidenció la formación de múltiples compuestos debido a las reacciones sucesivas de descomposición de la biomasa, dando lugar a una importante formación de compuestos solubles en la fase acuosa. El análisis FTIR de los residuos acuosos obtenidos después de secar la fracción acuosa obtenida presenta bandas características debidas a la presencia de compuestos oxigenados como aldehídos, ácidos carboxílicos, esteres y compuestos fenólicos formados durante la degradación de los carbohidratos y unidades estructurales de la biomasa lignocelulósica. Las principales bandas observadas en las fracciones acuosas obtenidas durante el desarrollo experimental, se muestran en la Tabla 7. En el caso de los espectros FTIR obtenidos para las fases acuosas provenientes de la licuefacción de Buchón de Agua, no se observó una tendencia en el aumento o disminución en las intensidades de las bandas características observadas con las condiciones de reacción evaluadas.

Tabla 8: Grupos funcionales identificados mediante FTIR en el residuo acuoso

\begin{tabular}{cll}
\hline $\mathrm{cm}^{-1}$ & \multicolumn{1}{c}{ Grupo funcional } & \multicolumn{1}{c}{ Tipo de compuesto } \\
\hline $3600-3300$ & Estiramiento $\mathrm{O}-\mathrm{H}$ & Agua, alcoholes y fenoles \\
$300-2850$ & Estiramiento $\mathrm{C}-\mathrm{H}$ de metil y metileno & Compuestos alifáticos: carbohidratos \\
$1680-1640$ & Estiramiento $-\mathrm{C}=\mathrm{C}-$ & Alquenos o ciclo alquenos \\
$1500-1400$ & Estiramiento C-C (en el anillo) & Compuestos aromáticos \\
$1400-1200$ & Deformación C-H & Carbohidratos \\
$1320-1000$ & Estiramiento C-O & Alcoholes, ácidos carboxílicos, ésteres y éteres \\
$950-910$ & Deformación O-H & Ácidos carboxílicos \\
$900-675$ & Deformación O-H & Compuestos aromáticos, fenoles \\
\hline
\end{tabular}

Al comparar las bandas características exhibidas en los espectros bajo las diferentes relaciones líquido/sólido empleadas $\left(1200,1300,1650-1700,1450 \mathrm{~cm}^{-1}\right)$, pudieron notarse algunas diferencias debido al aumento o disminución de algunas bandas, lo cual se debe a la solubilización en la fase acuosa de compuestos como monosacáridos, aldehídos, cetonas, ácidos carboxílicos, compuestos fenólicos y aminas aromáticas de bajo peso molecular, formados durante la descomposición de celulosa, hemicelulosa y lignina. La formación de estos productos está de acuerdo con las rutas de reacción propuestas por (Yin y Tan, 2012) para la licuefacción de material lignocelulósico. 


\section{CONCLUSIONES}

La evaluación del proceso de licuefacción hidrotérmica de Buchón de Agua permitió obtener un biocrudo con alta disponibilidad energética. El mayor rendimiento de biocrudo $(24,4 \%)$ se obtuvo a $300^{\circ} \mathrm{C}, 10 \mathrm{~g} / \mathrm{g}$ y $30 \mathrm{~min}$ de reacción, y el mayor poder calorífico $(37 \mathrm{MJ} / \mathrm{Kg})$ efectuando la licuefacción a $300^{\circ} \mathrm{C}, 15 \mathrm{~g} / \mathrm{g}$ con un tiempo de reacción de 0 min. En cuanto a la variable respuesta Rendimiento Energético, los mejores resultados se obtuvieron a $300^{\circ} \mathrm{C}, 60$ min y L/S=10 (experimento 15) con Rendimiento Energético de 6,34 MJ/Kg biomasa, a $300^{\circ} \mathrm{C}, 30 \mathrm{~min}$ y $\mathrm{L} / \mathrm{S}=10$ (experimento 5 ) con $\mathrm{RE}$ de $6,31 \mathrm{MJ} / \mathrm{Kg}$ biomasa y a $270^{\circ} \mathrm{C}, 60 \mathrm{~min}$ y $\mathrm{L} / \mathrm{S}=20$ (experimento 9) con 6,29 MJ/Kg biomasa, seleccionándose las condiciones del experimento 5 como las más adecuadas dada la menor relación L/S y el menor tiempo de reacción. El mejor ajuste al modelo de regresión empleado se obtuvo para la variable respuesta Rendimiento Energético con un 0,7730, con una significancia estadística para las variables de entrada Tiempo de reacción y Relación líquido/sólido. Se demostró asimismo la ventaja de adicionar catalizador a la reacción, al obtener un mayor Rendimiento Energético respecto al experimento de control Blanco bajo las mismas condiciones. Igualmente se pudo determinar que los biocrudos se componían principalmente de compuestos aromáticos, ácidos grasos, hidrocarburos alifáticos, cetonas, aldehídos y alcoholes, y ácidos carboxílicos a condiciones más drásticas.

\section{AGRADECIMIENTO}

Los autores agradecen el apoyo económico para el desarrollo de esta investigación, enmarcada dentro del proyecto Captura de $\mathrm{CO}_{2}$ por microalgas para la producción de biocombustibles financiado por Ruta N, la Universidad de Antioquia y Argos S.A.

\section{REFERENCIAS}

Anastasakis, K. y A. B. Ross, Hydrothermal Liquefaction of the Brown Macro-alga Laminaria saccharina: Effect of Reaction Conditions on Product Distribution and Composition, doi: 10.1016/J.BIORTECH.2011.01.031, Bioresource Technology, 102(7), 4876-4883 (2011)

Arteaga, J., W. Cuéllar y otros tres autores, Manejo de plantas acuáticas invasoras en embalses de EPM Caso: Buchón de Agua (Eichhornia crassipes) en el embalse de Porce II, Revista EPM, 3 (2010)

Biswas, B., R. Singh y otros tres autores, Pyrolysis of azolla, sargassum tenerrimum and water hyacinth for production of bio-oil, doi: 10.1016/j.biortech.2017.03.044, Bioresource Technology, 242, 139-145 (2017)

Caporgno, M.P., J. Pruvost y otros cuatro autores, Hydrothermal liquefaction of Nannochloropsis oceanica in different solvents, doi: 10.1016/j.biortech.2016.04.123, Bioresource Technology, 214, 404-410 (2016)

Demirbaş, A., Production of Biodiesel from Algae Oils, doi:10.1080/15567030701521775, Energy Sources, Part A: Recovery, Utilization, and Environmental Effects, 31(2), 163-168 (2008)

Duan, P., Z. Chang y otros cuatro autores, Hydrothermal Processing of Duckweed: Effect of Reaction Conditions on Product Distribution and Composition, doi: 10.1016/j.biortech.2012.08.106, Bioresource Technology, 135: 710-9 (2013)

Duan, P., B. Jin y otros seis autores, Thermo-chemical Conversion of Chlorella Pyrenoidosa to Liquid Biofuels, doi: 10.1016/j.biortech.2013.01.069, Bioresource Technology, 133, 197-205 (2013)

Duan, P. y P. E. Savage, Hydrothermal Liquefaction of a Microalga with Heterogeneous Catalysts, doi: 10.1021/ie100758s, Industrial \& Engineering Chemistry Research, 50 (1), 52-61 (2011)

Garcia-Perez, M., A. Chaala y otros tres autores, Characterization of bio-oils in chemical families, doi: 10.1016/j.biombioe.2006.02.006, Biomass and Bioenergy, 31 (4), 222-242 (2007)

Karagoz, S., T. Bhaskar, A. Muto e Y. Sakata, Comparative Studies of Oil Compositions Produced from Sawdust, Rice Husk, Lignin and Cellulose by Hydrothermal Treatment, doi: 10.1016/j.fuel.2005.01.004, Fuel, 84 (7-8), 875-884 (2005)

Kim, D., A. Koriakin y C. Lee, A parameter study for co-processing of petroleum vacuum residue and oil palm empty fruit bunch fiber using supercritical tetralin and decalin, doi: 10.1016/j.fuel.2016.05.034, Fuel, 181, 895-904 (2016)

Mazaheri, H., K.T. Lee y A. R. Mohamed, Influence of Temperature on Liquid Products Yield of Oil Palm Shell via Subcritical Water Liquefaction in the Presence of Alkali Catalyst, doi:10.1016/j.fuproc.2012.12.015, Fuel, Processing Technology, 110, 197-205 (2013)

Oasmaa, A., E. Kuoppala e Y. Solantausta, Fast Pyrolysis of Forestry Residue. 2. Physicochemical Composition of Product Liquid, doi: 10.1021/EF020206G, Energy Fuels, 17 (2), 433-443 (2003)

Osorio A., E. Gómez, D. Ocampo y L. Ríos, Licuefacción Solvotérmica de Madera de Acacia (Acacia mangium), http://dx.doi.org/10.4067/S0718-07642019000200211, Inf. Tecnol. 30(2), 211-222 (2019)

Rachel-Tang, D., A. Islam e Y. Taufiq-Yap, Bio-oil production via catalytic solvolysis of biomass, doi: 10.1039/C6RA27824H, RSC Adv., 7(13), 7820-7830 (2017) 
Singh, R., B. Balagurumurthy, A. Prakash y T. Bhaskar, Catalytic Hydrothermal Liquefaction of Water Hyacinth, doi: 10.1016/J.BIORTECH.2014.08.119, Bioresource Technology, 178, 157-165 (2015)

Tedesco, M., Algas en agua de consumo: acción humana y falta de manejo, Argentina Investiga (2009)

Xu, C. y J. Lancaster, Conversion of Secondary Pulp/Paper Sludge Powder to Liquid Oil Products for Energy Recovery by Direct Liquefaction in Hot-compressed Water, doi: 10.1016/j.watres.2007.11.007, Water Research, 42 (6-7), 1571-82 (2008)

Yang, X., H. Lyu y otros cuatro autores, Selective Extraction of Bio-oil from Hydrothermal Liquefaction of Salix psammophila by Organic Solvents with Different Polarities through Multistep Extraction Separation, doi: 10.15376/biores.9.3.5219-5233, BioResources, 9 (3), 5219-5233 (2014)

Yin, S. y Z. Tan, Hydrothermal Liquefaction of Cellulose to Bio-oil under Acidic, Neutral and Alkaline Conditions, doi: 10.1016/j.apenergy.2011.10.041, Applied Energy, 92, 234-239 (2012)

Yuan, X. Z., J. Y. Tong y otros tres autores, Comparative Studies of Products Obtained at Different Temperatures during Straw Liquefaction by Hot Compressed Water, doi: 10.1021/ef900027d, Energy Fuels, 23 (6), 3262-3267 (2009)

Zhang, J., W. T. Chen y otros tres autores, Hydrothermal Liquefaction of Chlorella pyrenoidosa in Sub- and Supercritical Ethanol with Heterogeneous Catalysts, doi:10.1016/j.biortech.2013.01.076, Bioresource Technology, 133C, 389-397 (2013)

Zhang, L., C. J. Li y otros tres autores, Hydrothermal Liquefaction of Water Hyacinth: Product Distribution and Identification, doi: 10.1080/15567036.2012.677937, Energy Sources, Part A: Recovery, Utilization, and Environmental Effects, 35 (14), 1349-1357 (2013)

Zhuang, Y., J. Guo y otros cuatro autores, Microwave-assisted Direct Liquefaction of Ulva prolifera for Bio-oil Production by Acid Catalysis, doi: 10.1016/j.biortech.2012.04.036, Bioresource Technology, 116, 133-9 (2012)

Zou, X., T. Qin y otros cuatro autores, Mechanisms and Main Regularities of Biomass Liquefaction with Alcoholic Solvents, doi: 10.1021/ef900590b, Energy \& Fuels, 23 (10), 5213-5218 (2009) 